\title{
Estudo das competências essenciais na atenção pré-natal: ações da equipe de enfermagem em Cuiabá, MT
}

O estudo teve como objetivo caracterizar as ações desenvolvidas pelos profissionais de enfermagem na assistência pré-natal no município de Cuiabá-MT. Foram realizadas observação não participante e entrevistas semi-estruturadas com 182 profissionais da equipe de enfermagem. A análise foi baseada nos documentos da Confederação Internacional de Parteiras e do Ministério da Saúde do Brasil. Identificou-se que a pré-consulta é feita pelo nível médio e as consultas de pré-natal pelos enfermeiros. As ações mais frequentes foram verificação da pressão arterial, do peso e anamnese, respectivamente em (100\%). Menos frequentes: inspeção das mucosas (28,3\%) e ausculta-cardiopulmonar $(9,4 \%)$. As competências essenciais no pré-natal foram desenvolvidas pela equipe de enfermagem, porém muitas delas apresentaram baixa frequência ou deixaram de ser realizadas em todas as consultas.

Descritores: Equipe de Enfermagem, Cuidado Pré-natal, Saúde da Mulher, Competência Profissional, Instituições de Saúde.

\section{Study of essential competences in prenatal attention: actions of the nursing team in Cuiaba}

The study aimed to characterize the actions developed by professionals in prenatal care in the city of Cuiabá-MT. Were performed non-participant observation and semi-structured interviews with 182 professional nursing staff. The analysis was based on documents of the International Confederation of Midwives and the Ministry of Health of Brazil. It was identified that the pre-appointment is made by high school and pre-natal consultations by nurses. The actions were more frequent checking of blood pressure, weight and medical history, respectively (100\%). Less common: inspection of the mucosa (28.3\%) and auscultation-cardiopulmonary (9.4\%). Core competencies in prenatal care were developed by the nursing staff, but many of them showed low frequency or were not accomplished in all queries.

Descriptors: Nursing Team, Prenatal Care, Women's Health, Professional Competence, Health Facilities.

\section{Estudio de las competencias essenciales en la atención prenatal: acciones del equipo de enfermeria en Cuiaba}

El estudio tuvo como objetivo caracterizar las acciones desarrolladas por profesionales en la atención prenatal en la ciudad de Cuiabá-MT. Se realizou la observación no participante y entrevistas semi-estructuradas con 182 profesionales de enfermería. El análisis se basó en los documentos de la Confederación Internacional de Matronas y el Ministerio de Salud de Brasil. Se identificó que la pre-designación se hace por los secundaristas y las consultas prenatales por las enfermeras. Las acciones más frecuente fueron la presión arterial, peso e anamnesis, respectivamente (100\%). Menos comunes: inspección de las mucosas $(28,3 \%)$ y la auscultación cardiopulmonar-(9,4\%). Las competencias básicas en la atención prenatal fueron desarrollados por el personal de enfermería, pero muchos de ellos mostraron baja frecuencia o no se realizo en todas las consultas.

Descriptores: Grupo de Enfermería, Atención Prenatal, Salud de la Mujer, Competencia Profesional, Istituciones de Salud.

\section{INTRODUÇÃO}

A situação da saúde feminina no período gravídico puerperal éuma preocupação aolongo de várias décadas e tem mobilizado tanto a sociedade civil organizada Iquanto o sistema governamental, na perspectiva de reduzir a mortalidade materna, que ainda constitui um desafio para o setor saúde e para a sociedade ${ }^{(1)}$.

Morte materna é definida pela Organização Mundial da Saúde $(\mathrm{OMS})^{(2)}$ como sendo o óbito de uma mulher grávida em qualquer fase da gestação até 42 dias após o parto, independentemente da duração e da localização da gravidez.

$\mathrm{Na}$ tentativa de reduzir a mortalidade materna e perinatal, o governo brasileiro incorporou, a partir de 1996, às políticas de atenção materna e infantil o projeto Maternidade Segura, em parceria com a Federação Brasileira das Sociedades de Ginecologia e Obstetrícia, o Fundo das Nações Unidas para a Infância e a
Organização Pan-Americana da Saúde ${ }^{(3)}$. E a principal meta de tal programa é a melhoria da assistência ao parto e nascimento.

Essa proposta encontra-se alicerçada na iniciativa do Grupo Interagências (IAG), formado pela OMS para estudos e propostas de redução da morte materna e perinatal em nível mundial. A iniciativa por uma maternidade sem risco, lançada em 1987 pela OMS como resultado dos estudos feitos pela IAG, propõe uma série de estratégias e passos bem definidos para aumentar a consciência mundial sobre o problema e proporcionar gravidez e parto mais seguros à mulher e aos recém-nascidos ${ }^{(4)}$.

Essas preocupações fundamentam-se no conhecimento sobre a magnitude do problema da morte materna em vários países, como no Brasil, e na convicção de que tal problema é reflexo da qualidade da assistência recebida pelas mulheres durante o ciclo gravídico-puerperal, pois a assistência pronta, oportuna e 
adequada pode evitar a maioria dessas mortes ${ }^{(5)}$.

Em 1997, o IAG convocou uma conferência internacional com o objetivo de examinar as lições aprendidas durante o primeiro decênio da iniciativa, identificar estratégias mais eficazes e mobilizar ações em nível nacional para implementar essas estratégias. Surgiu dessa conferência um claro consenso sobre o valor da atenção qualificada durante o parto, como uma intervenção fundamental para tornar as gravidezes e os partos mais seguros ${ }^{(4)}$.

A atenção qualificada ao pré-natal se refere ao processo pelo qual uma mulher grávida e o recém-nascido recebem atendimento adequado durante a gravidez, o trabalho de parto, o parto, o período pós-parto e o neonatal, independentemente do local de atendimento - no domicílio, no centro de saúde ou no hospital(4).

Nesse sentido, a Organização Mundial de Saúde (OMS), as Nações Unidas, a Confederação Internacional de Parteiras (ICM) e a Federação Internacional de Ginecologia e Obstetrícia (Figo) estabeleceram um consenso sobre a necessidade de uma clara definição sobre indicadores que norteassem o perfil esperado de um profissional com habilidades essenciais para oferecer a atenção à mulher no ciclo gravídico-puerperal(4).

Há evidência histórica e epidemiológica de que a atenção qualificada durante o parto e pós-parto tem um efeito significativo na redução das mortes maternas ${ }^{(6)}$. Os países que têm dado prioridade à ampliação da atenção qualificada durante o ciclo gravídico-puerperal têm alcançado uma redução substantiva na mortalidade materna ${ }^{(4,7)}$.

Tomando a relevância da temática e a contribuição da enfermagem na atenção qualificada ao pré-natal, este estudo buscou identificar, entre as ações desenvolvidas pelos profissionais de enfermagem no município de Cuiabá-MT, aquelas consideradas essenciais na atenção pré-natal que estão sendo desenvolvidas pelos profissionais de enfermagem em Cuiabá-MT.

Assim, o presente estudo teve por objetivo caracterizar as ações desenvolvidas pelos profissionais de enfermagem na assistência às mulheres grávidas na rede básica de saúde do município de Cuiabá-MT, tomando por referência as competências essenciais para a prática da obstetrícia da $\mathrm{ICM}^{(7) /}$ OMS/Figo e as ações propostas nos documentos oficiais pelo Ministério da Saúde do Brasil( ${ }^{(8)}$.

\section{METODOLOGIA}

Este estudo descritivo, transversal, de abordagem quantitativa, desenvolvido nas unidades de saúde de atenção básica da cidade de Cuiabá, capital do estado de Mato Grosso, de janeiro a dezembro de 2009, é parte da tese de doutorado intitulada "Estudo das competências essenciais na atenção pré-natal: ações da equipe de enfermagem no município de Cuiabá-MT", defendida em abril de 2010 na Universidade de São Paulo Escola de Enfermagem de Ribeirão Preto ${ }^{(9)}$.

$\mathrm{Na}$ ocasião da pesquisa, a população de Cuiabá era de aproximadamente 527 mil habitantes. O número de nascidos vivos em 2007 foi de 10.082, e a mortalidade geral hospitalar nesse mesmo ano foi de 1.966 pessoas. Dessas, 811 eram mulheres. A rede de saúde municipal contava com 63 equipes da Estratégia Saúde da Família (ESF), 22 Centros de Saúde (CS), oito policlínicas com atendimento 24 horas, seis policlínicas odontológicas, um hospital geral municipal de grande porte e um laboratório.

Compuseram o cenário do estudo 55 Unidades Básicas de Saúde da Família (UBSF) e 22 Centros de Saúde da rede municipal que prestam assistência ao pré-natal de baixo risco.

Foram considerados elegíveis para o estudo os profissionais de enfermagem que estivessem atuando na atenção pré-natal há pelo menos um mês no período de coleta de dados.

Participaram do estudo 182 profissionais de enfermagem, assim representados: 108 do nível médio, dos quais 60 são da ESF e 48 dos CS, e 74 enfermeiros, sendo 55 da ESF e 19 dos CS. Esses números correspondem ao quantitativo de profissionais que atuavam no prénatal, excluindo a perda de cinco enfermeiros da ESF e 13 dos CS.

Os critérios de exclusão foram: não atuar diretamente na assistência pré-natal, recusa expressa em participar do estudo ou a critério do pesquisador depois de, no mínimo, três tentativas sem sucesso para o agendamento da coleta dos dados.

Os procedimentos para a coleta dos dados ocorreram de duas formas: observação sistemática e não participante da atenção pré-natal oferecida pelos profissionais de enfermagem com auxilio de um check-list e entrevista semiestruturada com os mesmos profissionais que assistiram as gestantes durante as observações.

Os dados foram analisados utilizando o software Statistical Package for Social Sciences. Os resultados foram organizados através de estatística descritiva por meio de índices absolutos e porcentuais e apresentados em tabelas.

O projeto é parte da pesquisa"Atenção Qualificada ao Pré-Natal no Município de Cuiabá-MT", que foi aprovada pelo comitê de ética em pesquisas da Universidade Federal de Mato Grosso, pelo protocolo 598/2008. Solicitou-se a assinatura do Termo de Consentimento Livre e Esclarecido para os profissionais participantes e para as gestantes que permitiram a presença do pesquisador no ato do atendimento, cumprindo as exigências da resolução CNS 196/96.

\section{RESULTADOS}

O perfil dos profissionais levantado nas entrevistas revela uma predominância do sexo feminino, média de 36,5 anos para os enfermeiros e 43,3 anos para o nível médio, casados e com filhos, formados há mais de dez anos, com exceção das enfermeiras da Estratégia Saúde da Família. Não há enfermeiras com especialização em obstetrícia. A maioria dos profissionais referiu ter adquirido experiência em pré-natal no próprio trabalho e falta participação em cursos de atualização na área.

\section{Pré-consulta da gestante}

Observou-se 110 pré-consultas, sendo que 60 delas aconteceram 
na ESF e 50 nos CS. Entre as pré-consultas observadas, 105 foram realizadas pelos profissionais de nível médio e cinco pelos enfermeiros. Foi possível observar que os procedimentos de verificação da pressão arterial e do peso foram realizados em $100 \%$ das gestantes atendidas tanto nos CSs quanto nas unidades da ESF, No entanto, a medida da altura não ocorreu para todas as gestantes em ambos os serviços.

\section{Atenção pré-natal: a consulta de enfermagem}

Em 74 consultas de pré-natal realizadas por enfermeiros, 55 $(74,3 \%)$ aconteceram na ESF e $19(25,7 \%)$ nos CS. Todas as consultas realizadas durante o período de coleta de dados foram desenvolvidas por enfermeiros. $O$ tempo empregado em cada consulta, no período de observação, variou de oito a 50 minutos nas consultas de retorno e de 15 a 68 minutos nas consultas de primeira vez.

Com relação ao número da consulta, das 74 gestantes, $27(36,5 \%)$ estavam na primeira, $11(14,8 \%)$ na segunda, dez $(13,5 \%)$ na terceira e outras $26(35,2 \%)$ entre a quarta e a décima consulta. Verificou-se também que, das 27 gestantes de primeira consulta, $16(59,2 \%)$ estavam no primeiro trimestre e $11(40,8 \%)$ no segundo trimestre.

Na primeira consulta da gestante, além do cartão da gestante, os enfermeiros preenchem anotações no livro de registro do prénatal, formulários do Sisvan e do Sisprenatal e prontuário.

$\mathrm{Na}$ realização da anamnese das gestantes, a maioria dos enfermeiros iniciava pelo questionamento a respeito dos sinais e sintomas apresentados pelas gestantes. Das 74 gestantes, 48 $(64,8 \%)$ não apresentavam nenhuma queixa e, entre as outras 26 $(35,2 \%)$, as principais queixas foram: náuseas, vômito, cansaço, tontura, cefaleia, fraqueza, dor lombar, dor nas pernas, dor no baixo ventre, corrimento e prurido vaginal e disúria.

As principais informações coletadas pelos enfermeiros na anamnese são ilustradas na tabela 1. Na identificação, os itens que apareceram com mais frequência foram: o nome completo $(100 \%)$, a idade (100\%) e o endereço atual $(62,7 \%)$ das gestantes, especialmente as atendidas na ESF.

Em relação aos dados da gestação atual, foram levantados em frequência inferior a $90 \%$ das gestantes que estavam iniciando o pré-natal, com destaque para a data da última menstruação, e o cálculo da data provável do parto, respectivamente, em 85,1\% dos atendimentos. A idade gestacional foi estabelecida para $74 \%$ das gestantes de primeira vez e $89 \%$ para as demais gestantes. O conteúdo referente à sexualidade das 74 gestantes foi pouco explorado nas consultas de enfermagem em ambos os serviços.

Dos antecedentes de doenças familiares das gestantes verificados pelos enfermeiros, destacaram-se aquelas incluídas no cartão da gestante, sendo que a busca de algum familiar com diabetes mellitus ocorreu para $85 \%$ das mulheres grávidas, seguido da hipertensão arterial em $85 \%$ e da gemelaridade em $33,7 \%$ das 74 gestantes atendidas. Não foi verificada história familiar de doença de Chagas, nem se alguma das 74 gestantes teve contato sexual com portador do HIV.

Durante a coleta dos dados, pode-se observar que os enfermeiros verificaram os antecedentes obstétricos em 70,3\% das gestantes que estavam iniciando o pré-natal e em $16,4 \%$ das gestantes em consultas subsequentes. Das 74 gestantes, sete eram primigestas - não possuem antecedentes obstétricos. Entre os itens que permitem a avaliação dos antecedentes obstétricos das mulheres grávidas e que não foram checados com as gestantes estão: recém-nascidos com restrição do crescimento e/ou com má formação, nuliparidade ou multiparidade, pré-eclâmpsia ou eclâmpsia e a macrossomia fetal.

Em relação aos antecedentes pessoais das gestantes, a infecção urinária foi levantada em $81 \%$ delas, além da hipertensão arterial e a diabetes mellitus em $82 \%$. Os enfermeiros não investigaram entre as gestantes doenças como as endocrinopatias, malária e viroses.

Quanto aos dados socioeconômicos, foram checados: a escolaridade em $53,7 \%$, o estado conjugal em $62,4 \%$ e, ainda, a profissão em $32,5 \%$ dos atendimentos. A renda familiar, pessoas da família com renda, condições de moradia, condições de saneamento, número e idade dos dependentes e a distância percorrida da residência da gestante até o serviço de saúde foram informações que deixaram de ser investigadas.

A abordagem dos antecedentes ginecológicos das 74

Tabela 1 - Frequência do conteúdo da anamnese de 74 gestantes atendidas por enfermeiros na rede básica de saúde em Cuiabá-MT. Cuiabá, 2009.

\begin{tabular}{|c|c|c|c|c|}
\hline \multirow[t]{2}{*}{ Anamnese } & \multicolumn{2}{|c|}{$\begin{array}{l}\text { Primeiras consultas } \\
n=27\end{array}$} & \multicolumn{2}{|c|}{$\begin{array}{l}\text { Consultas subsequentes } \\
\qquad n=55\end{array}$} \\
\hline & $\mathrm{n}^{\circ}$ & $\%$ & $n^{\circ}$ & $\%$ \\
\hline Identificação & 27 & 100 & 36 & 65,4 \\
\hline Dados da gestação atual & 23 & 85,1 & 33 & 60,0 \\
\hline Antecedentes familiares & 23 & 85,1 & 9 & 16,3 \\
\hline Antecedentes Obstétricos & 19 & 70,3 & 9 & 16,3 \\
\hline Antecedentes pessoais & 22 & 81,4 & 7 & 12,7 \\
\hline Dados socioeconômicos & 20 & 74,0 & 5 & 10,6 \\
\hline Antecedentes Ginecológicos & 9 & 33,3 & 3 & 5,5 \\
\hline Risco gestacional & 9 & 33,3 & 2 & 3,6 \\
\hline
\end{tabular}


gestantes ficou restrita às cirurgias ginecológicas realizadas, sendo investigada em apenas $12(16,2 \%)$ das gestantes atendidas. Outras informações que apresentaram menor frequência foram: a data do último teste de Papanicolau, alterações nas mamas, doença inflamatória pélvica, regularidade dos ciclos menstruais, doenças sexualmente transmissíveis e uso de anticoncepcionais.

Entre as intercorrências clínicas e crônicas que devem ser consideradas na avaliação do risco gestacional, a infecção urinária foi a mais frequente pesquisada pelos enfermeiros em ambos os serviços, representando a investigação em nove $(12,1 \%)$ das mulheres grávidas que fizeram consulta pré-natal. Observou-se que, entre as variáveis relacionadas à história clínica da gestante e que não foram avaliadas pelos enfermeiros, estavam as pneumopatias, nefropatias, hemopatias, epilepsia e as doenças autoimunes.

Outras situações consideradas pela Confederação Internacional de Parteiras e pelo Ministério da Saúde como risco gestacional e que não foram avaliadas são: desvio do crescimento uterino, número de fetos, condições do líquido amniótico, trabalho de parto prematuro e gravidez prolongada, amniorrexe prematura e isoimunização.

Tabela 2 - Frequências dos procedimentos realizados no exame físico geral de 74 gestantes atendidas por enfermeiros da rede básica de saúde do município de Cuiabá-MT. Cuiabá, 2009.

\begin{tabular}{|l|c|c|c|c|c|c|}
\multicolumn{1}{|c|}{ Exame físico geral } & \multicolumn{2}{c|}{$\begin{array}{c}\text { CS } \\
n=19\end{array}$} & \multicolumn{2}{c|}{$\begin{array}{c}\text { USF } \\
n=55\end{array}$} & \multicolumn{2}{c|}{$\begin{array}{c}\text { TOTAL } \\
n=74\end{array}$} \\
\hline Análise nutricional & $n^{\circ}$ & $\%$ & $n^{\circ}$ & $\%$ & $n^{\circ}$ & $\%$ \\
Exame dos membros inferiores & 4 & 21,1 & 43 & 78,1 & 47 & 63,5 \\
Pesquisa de edema & 4 & 21,1 & 30 & 54,5 & 34 & 45,9 \\
Inspeção da pele e mucosas & 4 & 21,1 & 30 & 54,5 & 34 & 45,9 \\
Palpação: pescoço e axila & 5 & 26,3 & 16 & 29,1 & 21 & 28,3 \\
Ausculta cárdio-pulmonar & 2 & 10,5 & 9 & 16,4 & 11 & 14,8 \\
Frequência cardíaca & 0 & 0 & 7 & 12,7 & 7 & 9,4 \\
& 1 & 5,3 & 5 & 9,1 & 6 & 8,1 \\
\hline
\end{tabular}

Em relação aos procedimentos que foram realizados no exame físico geral no pré-natal, a tabela 2 revela que menos de $50 \%$ das gestantes foram examinadas.

O exame de MMII e a pesquisa de edema foram realizados em $45,9 \%$ do total das consultas. A ausculta cardíaca e pulmonar não fez parte dos procedimentos realizados no exame físico pela maioria dos enfermeiros. Os procedimentos realizados pelos enfermeiros durante o exame físico das gestantes, durante o período de observação, evidenciam lacunas nessa etapa do processo de enfermagem, visto que a inspeção, palpação, percussão e ausculta das estruturas corporal foram etapas não contempladas nas consultas observadas.

Os procedimentos realizados no exame físico obstétrico, ilustrados na tabela 3, revelam que menos de $50 \%$ das gestantes foram submetidas a ele.

A frequência da medida da altura uterina foi realizada em
Tabela 3 - Frequência dos procedimentos realizados no exame físico obstétrico nas consultas de prénatal de 74 gestantes atendidas por enfermeiros da rede básica de saúde do município de Cuiabá-MT. Cuiabá, 2009.

\begin{tabular}{|l|c|c|c|c|c|c|}
\hline \multicolumn{1}{|c|}{ Exame obstétrico } & \multicolumn{2}{c|}{$\begin{array}{c}\text { CS } \\
n=19\end{array}$} & \multicolumn{2}{c|}{$\begin{array}{c}\text { USF } \\
n=55\end{array}$} & \multicolumn{2}{c|}{$\begin{array}{c}\text { TOTAL } \\
n=74\end{array}$} \\
\hline Medida da altura uterina & $\mathrm{n}^{\circ}$ & $\%$ & $\mathrm{n}^{\circ}$ & $\%$ & $\mathrm{n}^{\circ}$ & $\%$ \\
Aferição dos BCF & 4 & 21,1 & 32 & 58,2 & 36 & 48,6 \\
Exame das mamas & 4 & 21,1 & 30 & 54,5 & 34 & 45,9 \\
Palpação obstétrica & 5 & 26,3 & 24 & 43,6 & 29 & 39,1 \\
Inspeção dos genitais externos & 3 & 15,8 & 19 & 34,5 & 22 & 29,7 \\
Exame especular & 0 & 0 & 1 & 1,8 & 1 & 1,3 \\
\hline & 0 & 0 & 1 & 1,8 & 1 & 1,3 \\
\hline
\end{tabular}

$48,6 \%$ das consultas, seguida da ausculta do BCF $(45,9 \%)$. Vale destacar que, em duas unidades, não havia aparelho de sonar ou estetoscópio de Pinard. O exame clínico das mamas foi realizado em $39,1 \%$ das gestantes, enquanto a palpação, procedimento para verificar a posição e apresentação fetal, foi executada em $29,7 \%$ das gestantes. Foi possível constatar também que, nas instituições estudadas, durante o período de observação, apenas um profissional realizou a inspeção dos genitais e, embora tenha utilizado espéculo vaginal no exame, não foi procedida a coleta de Papanicolau. Durante as observações, constatou-se que um profissional de nível médio verificou a altura uterina e aferiu os batimentos cardiofetais com o uso do aparelho Doppler, na presença do enfermeiro.

Em relação às orientações ofertadas às gestantes, as que mais de destacaram foram: local de desfecho da gravidez $(56,7 \%)$, alimentação $(43,2 \%)$ e agendamento das consultas e exames (39,1\%). Destaca-se que os enfermeiros da ESF foram os que mais orientaram as gestantes durante as consultas observadas.

A solicitação de exames laboratoriais e de diagnóstico feita pelos enfermeiros foi o seguinte: grupo sanguíneo, fator $\mathrm{Rh}$, hemograma, glicemia, urina tipo I, parasitológico de fezes, teste anti-HIV, sorologias (hepatite A, hepatite B, hepatite C, rubéola, sífilis, toxoplasmose, citomegalovírus e clamídia), coombs indireto e lipidograma. Um enfermeiro solicitou exames (Baar e cultura de escarro) para monitoração do tratamento da tuberculose em uma gestante. Essa gestante já estava em acompanhamento pré-natal de alto risco e frequentava, também, a unidade de saúde da família.

Com relação à ultrassonografia requisitada pelos enfermeiros para $10,8 \%$ das gestantes, os mesmos preencheram o pedido do exame e quem o assinava era o médico da unidade. Constatou-se, também, que nos dois modelos estudados a coleta de material para exame colpocitológico não foi efetuada em nenhuma consulta durante o período de coleta de dados.

Quanto aos medicamentos prescritos pelos enfermeiros, o sulfato ferroso e ácido fólico o foram em maior frequência, enquanto que o Buscopan, Paracetamol e Plasil tiveram 
menor frequência de prescrição. Os enfermeiros dos CS não prescrevem medicamentos rotineiramente. Observou-se que a prescrição de medicamentos por enfermeiros em Cuiabá ainda não está claramente definida e, diante de alguma gestante com queixa clínica, a maioria dos enfermeiros encaminha para avaliação e conduta do médico.

As demais atividades realizadas pelos enfermeiros durante a consulta foram: encaminhamento para vacina antitetânica $(29,7 \%)$, encaminhamento para Papanicolou $(9,4 \%)$, agendamento das consultas subsequentes $(40,5 \%)$ e anotações no prontuário e cartão da gestante (72,9\%).

Do total de 74 gestantes consultadas, 35 estavam com vacina antitetânica em dia. A anotação/registro no prontuário e cartão da gestante foi efetuada em $72,9 \%$ das consultas. Ressalta-se que todas as gestantes portavam o cartão da gestante no momento da consulta. O agendamento das consultas subsequentes foi orientado em $40,5 \%$ do total das gestantes. As consultas subsequentes são agendadas na recepção das unidades estudadas, e constatou-se que muitas das gestantes já conheciam a rotina desse agendamento. Com relação ao encaminhamento para o exame de Papanicolau, apenas 9,4\% das gestantes foram orientadas a fazê-lo. Durante a coleta dos dados, ficou evidente que a realização do exame Papanicolau é assunto controverso entre os enfermeiros de Cuiabá: há aqueles que recomendam e outros que contraindicam tal exame no período gestacional, mesmo para gestantes com queixa ginecológica, justificando que tal exame pode causar aborto.

Constatou-se que os enfermeiros encaminharam $6,7 \%$ das gestantes para avaliação e conduta médica, frente a queixas ginecológicas de tais gestantes (dor no baixo ventre, suspeita de perda sanguínea e leucorreia). Observou-se, também, que, das 74 gestantes atendidas, três (4\%) e duas $(2,7 \%)$ foram encaminhadas para avaliação odontológica e nutricional, respectivamente.

Quanto à educação em saúde, observou-se, durante a coleta dos dados, que houve ações educativas em apenas três unidades da ESF, duas ocorreram na sala de espera e uma na sala de reuniões. Em relação ao acolhimento, na maioria das vezes, foi realizado durante as consultas.

\section{DISCUSSÃO}

Para que o pré-natal na rede básica de saúde de CuiabáMT seja qualificado, há necessidade da (re)organização das ações a serem desenvolvidas pela equipe de enfermagem, tendo em vista que os procedimentos propostos pela International Confederation of Midwives ${ }^{(7)}$ e pelo Ministério da Saúde brasileiro ${ }^{(8)}$ apresentaram baixa frequência, e alguns deles não foram realizados. Além disso, identificaram-se algumas fragilidades que comprometem a atenção qualificada como a situação na qual se observou o técnico de enfermagem realizando procedimentos para os quais não possui habilitação, incluindo a aferição da altura uterina e dos batimentos cardiofetais. A realização de tais procedimentos insere-se na consulta de enfermagem no pré-natal, cuja ação para a equipe de enfermagem é restrita aos enfermeiros, os quais são legalmente reconhecidos pela lei do exercício profissional $n^{\circ} 7498$, que explicita que a realização do pré-natal de baixo risco é uma das atribuições do enfermeiro ${ }^{(10)}$.

Os resultados mostraram que não há definição de condutas estabelecidas em protocolos assistenciais. Da mesma forma, ficou evidente que as ações desenvolvidas na assistência ao pré-natal pela equipe de enfermagem em Cuiabá-MT não são padronizadas e orientadas por práticas baseadas em evidências científicas, comprometendo, assim, a atenção qualificada ao pré-natal.

Os padrões e protocolos definem o tipo de cuidado oferecido em cada nível do sistema de saúde e a contribuição de cada categoria de provedor. Os mesmo são essenciais para orientar e apoiar a prática da atenção qualificada no ciclo gravídico puerperal, assim como para avaliar e melhorar o desempenho dos provedores ${ }^{(11)}$. Da mesma forma, as normas e diretrizes também podem ser utilizadas como base para autoavaliação na prática clínica por parte do pessoal qualificado e para avaliações de programas de supervisão por pares ${ }^{(12-13)}$.

Contudo, quando se pretende melhorar a capacidade dos profissionais para proteger e promover a saúde materna, infantil e comunitária, é indispensável estabelecer programas de capacitação de alta qualidade e de supervisão(14). Chama-se a atenção para que um ambiente favorável ao desenvolvimento de pessoas capacitadas implica, entre outras coisas, a existência de associações profissionais que promovam e defendam uma atenção de alta qualidade durante a gestação(4).

Nessa direção, a Associação Brasileira de Obstetrizes e Enfermeiras Obstétricas (Abenfo) tem exercido papel importante na elaboração de políticas nacionais de formação e atenção à mulher e de normas técnicas baseadas em evidência científicas e de perfis de competências básicas no atendimento à mulher e ao neonato para enfermeiros e obstetrizes $^{(8)}$.

\section{CONCLUSÃO}

A limitação deste estudo está na avaliação de competências, considerando que não se buscou avaliar o desempenho dos profissionais acerca das competências desenvolvidas na obstetrícia pela equipe de enfermagem do município de Cuiabá-MT, mas sim analisar se as ações relacionadas àquelas competências eram desenvolvidas no atendimento às gestantes. 
A realização deste estudo permitiu identificar que, em Cuiabá-MT, o pré-natal conta com a participação efetiva dos profissionais da enfermagem que atuam nas unidades de saúde da atenção básica, que são os Centros de Saúde e as Unidades Básicas de Saúde da Família.

As competências essenciais para a assistência pré-natal foram desenvolvidas pela equipe de enfermagem. No entanto, muitas delas apresentaram baixa frequência ou deixaram de ser realizadas em todas as consultas. A ausência de protocolos que orientem as condutas dos profissionais de enfermagem na atenção ao pré-natal é um dos fatores que comprometem o desenvolvimento de tais competências.

As atividades realizadas com maior frequência no pré-natal foram a verificação da pressão arterial e do peso, anamnese, verificação da data da última menstruação, cálculo da data provável do parto e da idade gestacional, exame dos membros inferiores, pesquisa de edemas, ausculta dos batimentos cardiofetais, medida da altura uterina, orientações (local onde é feito o parto, alimentação, agendamento de consultas de retorno e dos exames), solicitação e interpretação de exames laboratoriais, agendamento das consultas de retorno e anotações no prontuário e no cartão da gestante.

As ações e procedimentos que apresentaram menor frequência foram ausculta cardiopulmonar, verificação da frequência cardíaca, inspeção de pele e mucosas, palpação do pescoço e axila, exame das mamas, palpação obstétrica, inspeção dos genitais externos, exame especular, orientações (sexualidade, higiene, uso de preservativos, atividade laboral, cuidados com as mamas, aleitamento materno, planejamento familiar), ações educativas, prescrição de medicamentos, retorno para o puerpério, encaminhamento para sala de vacina, dentista e nutricionista e retorno para a consulta de puerpério.

As ações e os procedimentos não realizados durante a coleta dos dados relacionam-se às atividades em grupo de gestantes e a classificação do risco gestacional, para as quais o município não possui protocolo, o que compromete a atenção qualificada ao pré-natal pelos enfermeiros.

\section{Referências}

1. Duarte SJH, Andrade SMO. Assistência pré-natal no Programa Saúde da

Familia, 2006. Rev Enferm. 2006;10:121-5.

2. Organização Mundial da saúde. Maternidade segura. Assistência ao parto

normal: um guia prático. Genebra: OMS; 1996.

3. Ministério da Saúde (BR). Federação Brasileira das Sociedades de Ginecologia

e Obstetrícia. Associação Brasileira de Obstetrizes e Enfermeiras Obstetras. Parto,

aborto e puerpério: assistência humanizada à mulher. Brasília: Ministério da

Saúde; 2003.

4. Macdonald M, Starrs A. La atencion calificada durante el parto: un cuaderno

informativo para salvar la vida de las mujeres y mejorar la salud de los recién

nacidos. New York: Family Care Internacional; 2003.

5. Tanaka ACd'A. Dossiê mortalidade materna. Rede Saúde. 2001.

6. Moura ERF, Silva RM. Competência profissional e assistência em anticoncepção.

Rev Saúde Pública. 2005;39:795-801.

7. International Confederation of Midwives. Essentiel competencies for basic

midwifery practice 2002. New York: ICM; 2002.

8. Ministério da Saúde (BR). Pré-natal e puerpério. Atenção qualificada e

humanizada. Brasília: Ministério da Saúde; 2006.

9. Duarte SJH. Estudo das competências essenciais na atenção pré-natal: açōes da equipe de enfermagem no município de Cuiabá, MT [tese]. Ribeirão Preto: Universidade de São Paulo, Escola de Enfermagem de Ribeirão Preto; 2010. 10. Presidência da República Federativa do Brasil. Lei nº. 7.498, de 25 de jun. de 1986. Dispōe sobre a regulamentação do exercício da

Enfermagem e dá outras providências. Diário Oficial da União. Brasília, 26 jun 1986, seção I p. 9.273-9275.

11. Kak N, Burkhalter B, Cooper MA. Measuring the competence of healthcare providers. Operations research issue paper, Bethesda, Agency for International Development (USAID) by the Quality Assurance (QA) Project [Internet]. [citado em 2009 Ago 12]. Disponível em: http://www.qaproject.org/pubs/PDFs/competence.pdf 12. Mamede MV, Dotto LMG, Oliveira ME, Bruggemann O, Riesgo MLG, Gualda DMR, Arantes SL. Minuta do Concurso para Certificação e Titulação em Enfermagem Obstétrica e Neonatal pela Associação Brasileira de Enfermagem Obstétrica e Neonatal. Rio de Janeiro: [s.n.]; 2008.

13. Lourenção DCA, Bentido GAV. Competências gerenciais na formação do enfermeiro. Rev Bras Enferm. 2010;63(1):91-7.

14. Shimizu HE, Lima MG. As dimensões do cuidado pré-natal na consulta de enfermagem. Rev Bras Enferm. 2009;62(3):387-92. 